\title{
Activation of CD95L fusion protein prodrugs by tumor-associated proteases
}

\author{
I Watermann ${ }^{1}$, J Gerspach ${ }^{1}$, M Lehne ${ }^{2}$, J Seufert ${ }^{2}$, B Schneider ${ }^{1}$, K Pfizenmaier ${ }^{\star, 1,3}$ and H Wajant ${ }^{\star, 2,3}$
}

To achieve tumor cell-restricted activation of CD95, we developed a CD95L fusion protein format, in which CD95L activity is only unmasked upon antibody-mediated binding to tumor cells and subsequent processing by tumor-associated proteases, such as matrix metalloproteases (MMPs) and urokinase plasminogen activator (UPA). On target-negative, but MMP- and uPA-expressing HT1080 tumor cells, the CD95L prodrugs were virtually inactive. On target antigen-expressing HT1080 cells, however, the CD95L prodrugs showed an apoptotic activity comparable to soluble CD95L artificially activated by crosslinking. CD95 activation by the CD95L prodrugs was preceded by prodrug processing. Apoptosis was blocked by inhibitors of MMPs or uPA and by neutralizing antibodies recognizing the targeted cell surface antigen or the CD95L moiety of the prodrugs. In a xenotransplantation tumor model, local application of the prodrug reduced the growth of target antigen-expressing, but not antigen-negative tumor cells, verifying targeted $\mathrm{CD} 95 \mathrm{~L}$ prodrug activation in vivo.

Cell Death and Differentiation (2007) 14, 765-774. doi:10.1038/sj.cdd.4402051; published online 20 October 2006

The CD95L-CD95 system has a complex role in tumor biology. For example, it has been shown that upregulation of CD95 and CD95L by chemotherapeutic drugs can significantly contribute to the antitumoral effects of such reagents and in several experimental models, tumor-localized expression of CD95L boosts antitumoral immunity. ${ }^{1}$ Vice versa, there is evidence that CD95-resistant tumors use CD95L to fight tumor infiltrating T cells. ${ }^{2}$ Moreover, recent studies revealed a strong capacity of CD95 to engage proinflammatory pathways such as the NF- $\kappa$ B pathway and the MAP kinase cascades. ${ }^{3}$ These pathways regulate cell survival, proliferation, angiogenesis and cell migration. It is therefore conceivable that non-apoptotic CD95 signaling exerts protumoral effects in apoptosis-resistant tumor cells. Exploitation of the antitumoral effects of CD95 by exogenous delivery of CD95L or agonistic antibodies is hampered so far by severe systemic toxicity, foremost owing to massive apoptosis induction in hepatocytes, resulting in acute liver failure. ${ }^{4}$ To overcome this limitation, solutions have to be found that allow tumorlocalized activation of CD95. The most common way to achieve tumor-restricted action is the use of antibodies, especially recombinant antibody fragments, as targeting devices. The tumor-associated activity of antibody conjugates of effector molecules, ranging from cytokines over toxins to radioactive isotopes, primarily relies on the enrichment of these conjugates in the tumor by antibody-mediated binding to the corresponding tumor-associated antigen. As the effector molecules typically used for the construction of antitumoral antibody conjugates are active independent of antigen recognition, such compounds still may exert considerable systemic activity. A notable exception from this are antibodyCD95L fusion proteins, because soluble, homotrimeric forms of CD95L are poorly active. ${ }^{5}$ Therefore, the high tumorselective activity of trimeric antibody-CD95L fusion proteins primarily is not due to antibody-mediated tumor trapping, but rather relies on targeting associated conversion of poorly active soluble CD95L into a highly active cell surface attached entity with membrane CD95L-like activity. ${ }^{6}$ However, this highly tumor-selective activity of antibody-CD95L fusion proteins diminishes when the cell surface antigen used for targeting/activation is not exclusively expressed in the tumor or when the antibody domain used for construction of the fusion protein displays an intrinsic tendency to autoaggregate. ${ }^{6}$ In the latter case, this will cause secondary aggregation of the trimeric CD95L fusion protein to multimeric protein complexes, evoking antigen-independent restoration of CD95L activity. To circumvent these problems, a CD95L prodrug concept appears necessary in which tumor-associated restoration of CD95L activity is achieved by additional, targeting independent mechanisms. As an example, we recently demonstrated the construction of a TNF prodrug, which displayed greater than 1000-fold increase in specific TNF activity upon cell membrane targeting and processing by tumor-associated metalloproteases such as matrix metalloprotease (MMP) 2. ${ }^{7}$ TNF prodrugs consist of a cell surface tumor antigen recognizing antibody-TNF fusion protein, which

\footnotetext{
${ }^{1}$ Institute of Cell Biology and Immunology, University of Stuttgart, Stuttgart, Germany and ${ }^{2}$ Department of Molecular Internal Medicine, Medical Clinic and Polyclinic II, University of Wuerzburg, Wuerzburg, Germany

${ }^{*}$ Corresponding authors: H Wajant, Department of Molecular Internal Medicine, Medical Clinic and Polyclinic II, University of Wuerzburg, Roentgenring 11, Wuerzburg 97070, Germany. Tel: + 4993120171882 ; Fax: + 4993120171070 ;

E-mail: harald.wajant@mail.uni-wuerzburg.de

or K Pfizenmaier, Institute of Cell Biology and Immunology, University of Stuttgart, Allmandring 31, Stuttgart 70569, Germany.

E-mail: klaus.pfizenmaier@izi.uni-stuttgart.de

${ }^{3}$ These authors contributed equally to this work.

Keywords: apoptosis; CD95L; prodrug; proteases

Abbreviations: MMP, matrix metalloprotease; UPA, urokinase plasminogen activator; FAP, fibroblast activation protein

Received 23.3.06; revised 16.8.06; accepted 08.9.06; Edited by DR Green; published online 20.10.06
} 
is carboxy-terminally extended with a TNF inhibitory domain composed of the extracellular domain of TNF receptor-1. As the inhibitory domain was linked by a protease-sensitive linker to the antibody-TNF domain, the inhibitory domain was released upon proteolytic processing by membrane-associated MMP2 or related proteases when the construct was targeted to tumor cells via its specific antibody module. ${ }^{7}$ As even small carboxy-terminal extensions of CD95L, comprising just a few amino acids, strongly reduced CD95L activity in CD95L fusion proteins, we developed a prodrug format in which the authentic CD95L carboxy-terminus is preserved. In this prodrug format, the inhibitory domain was placed aminoterminally to the antibody-CD95L module, separated by a protease sensitive motif. To strengthen the inhibitory effect of the extracellular domain of CD95 in the CD95L prodrug, a trimerization domain was also included. We here describe the functional properties of such CD95L prodrugs and demonstrate their activation upon selective processing on targetpositive tumor cells in vitro and in vivo.

\section{Results and Discussion}

Generation of a FAP-specific CD95L prodrug. In a first attempt to construct a CD95L prodrug that becomes selectively activated by tumor-associated proteases, we used the domain architecture of recently published TNF prodrugs. ${ }^{7,8}$ In the corresponding CD95L prodrug, named SC40-CD95L-PL-CD95, an amino-terminal scFv (SC40), recognizing the tumor stroma marker fibroblast activation protein (FAP), ${ }^{9,10}$ is followed by the extracellular domain of $\mathrm{CD} 95 \mathrm{~L}$, a protease-sensitive linker containing repeats of the consensus recognition sequence for MMP2 and related proteases (PL) as well as a carboxy-terminal inhibitory domain composed of the extracellular domain of CD95. To ease purification and analysis, we introduced a Flag tag between the single chain and the CD95L domain of this and

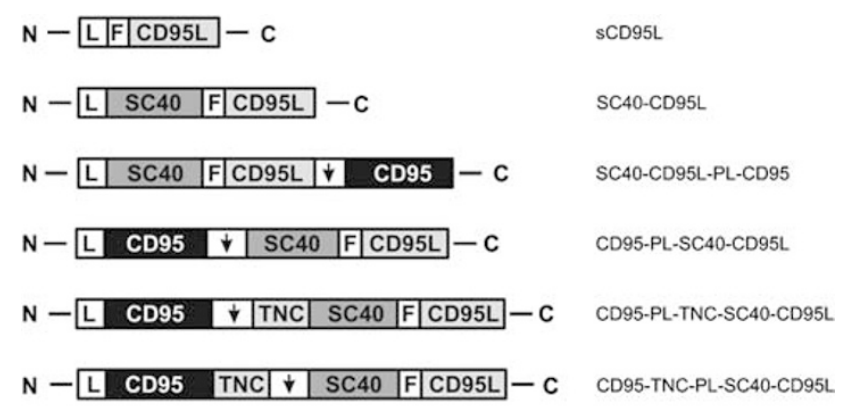

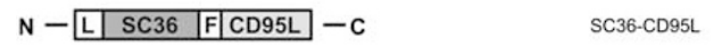

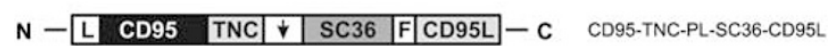

Figure 1 Scheme of the CD95L fusion proteins used in this study. L: leader peptide, SC40: human FAP-specific single-chain antibody fragment '40', SC36: human and mouse FAP-specific single-chain antibody fragment '36', F: Flag tag, CD95L: human CD95L (aa 139-281), CD95: human CD95 (aa 15-173), TNC: chicken tenascin aa (110-139), arrow: MMP2-sensitive linker all other prodrug-related molecules investigated in this study (Figure 1). Of course, for an intended clinical use, the Flag tag would be omitted to reduce potential antigenicity of the prodrug. However, each genetically engineered protein formally cannot rule out the creation of antigenic neoepitopes, in our case potentially caused by the specific new arrangement of naturally occurring domains into the prodrug molecule. A potential immune response towards the prodrug, however, can only be revealed in the course of clinical trials.

SC40-CD95L-PL-CD95 (Figure 1) was analyzed on FAPnegative HT1080 cells and FAP-positive HT1080 cells which are comparably sensitive for CD95-mediated apoptosis, but are differentially killed by SC40-CD95L (Figure $2 a$ and b). SC40-CD95L-PL-CD95 showed no apoptotic activity, neither on FAP-negative HT1080 cells nor on FAP-positive HT1080 cells (Figure 2c), on which the corresponding TNF prodrug was activated with high efficiency. ${ }^{7}$ A CD95L fusion protein (SC40-CD95L-PL) which corresponded in structure to the completely processed CD95L prodrug, that is comprising a Cterminal addition of four amino acids corresponding to a proteolytically cleaved MMP linker, ${ }^{7}$ was found to possess only marginal bioactivity after secondary crosslinking (data not shown) suggesting that an authentic carboxy-terminus is more important for CD95L function than for TNF function. We therefore constructed a CD95L prodrug in which the CD95L carboxy-terminus was preserved. In this prodrug (CD95-PLSC40-CD95L), the inhibitory extracellular domain of CD95 preceded the single chain-CD95L domain. CD95-PL-SC40CD95L was comparatively active as SC40-CD95L on FAPexpressing HT1080 cells, but unexpectedly was also highly active on FAP-negative parental HT1080 cells after secondary crosslinking (Figure $2 \mathrm{~d}$ ). The latter observation indicated that the CD95L module of the CD95-PL-SC40-CD95L fusion protein was not or only inefficiently blocked by the inhibitory CD95 domain of the molecule. Indeed, it has been shown that an Ig Fc-fusion protein of the extracellular domain of CD95 is a rather weak neutralization reagent for CD95L. ${ }^{11}$ Noteworthy, it has also been shown that the poor neutralizing capacity of the extracellular domain of CD95 can be significantly enhanced by enforced multimerization with the pentamerization domain of cartilage oligomeric matrix protein ${ }^{11}$ or with the trimerization domain of the cartilage matrix protein (CMP). ${ }^{11}$ In order to enhance the neutralization capacity of the CD95 domain in our CD95L prodrug, we introduced the trimerization domain of tenascin-C (TNC) ${ }^{12}$ between the inhibitory CD95 domain and the SC40-CD95L module. In one variant, the trimerization domain of TNC was immediately placed after the CD95 domain (CD95-TNC-PL-SC40-CD95L) and in a second variant the TNC trimerization domain was placed between the protease-sensitive linker and the scFv domain (CD95-PLTNC-SC40-CD95L), (Figure 1). Both CD95L prodrugs were almost as active as SC40-CD95L or secondarily crosslinked CD95L on FAP-expressing cells (Figure $2 \mathrm{e}$ and $\mathrm{f}$ ). More important, both variants were poorly active on FAP-negative cells and remained significantly less active compared to SC40-CD95L even after crosslinking (Figure $2 e$ and f). The markedly reduced activity of the crosslinked fusion proteins on target-negative cells confirmed that indeed the CD95 inhibitory domain sufficiently blocked the action of the CD95L domain. As the CD95-TNC-PL-SC40-CD95L prodrug showed 

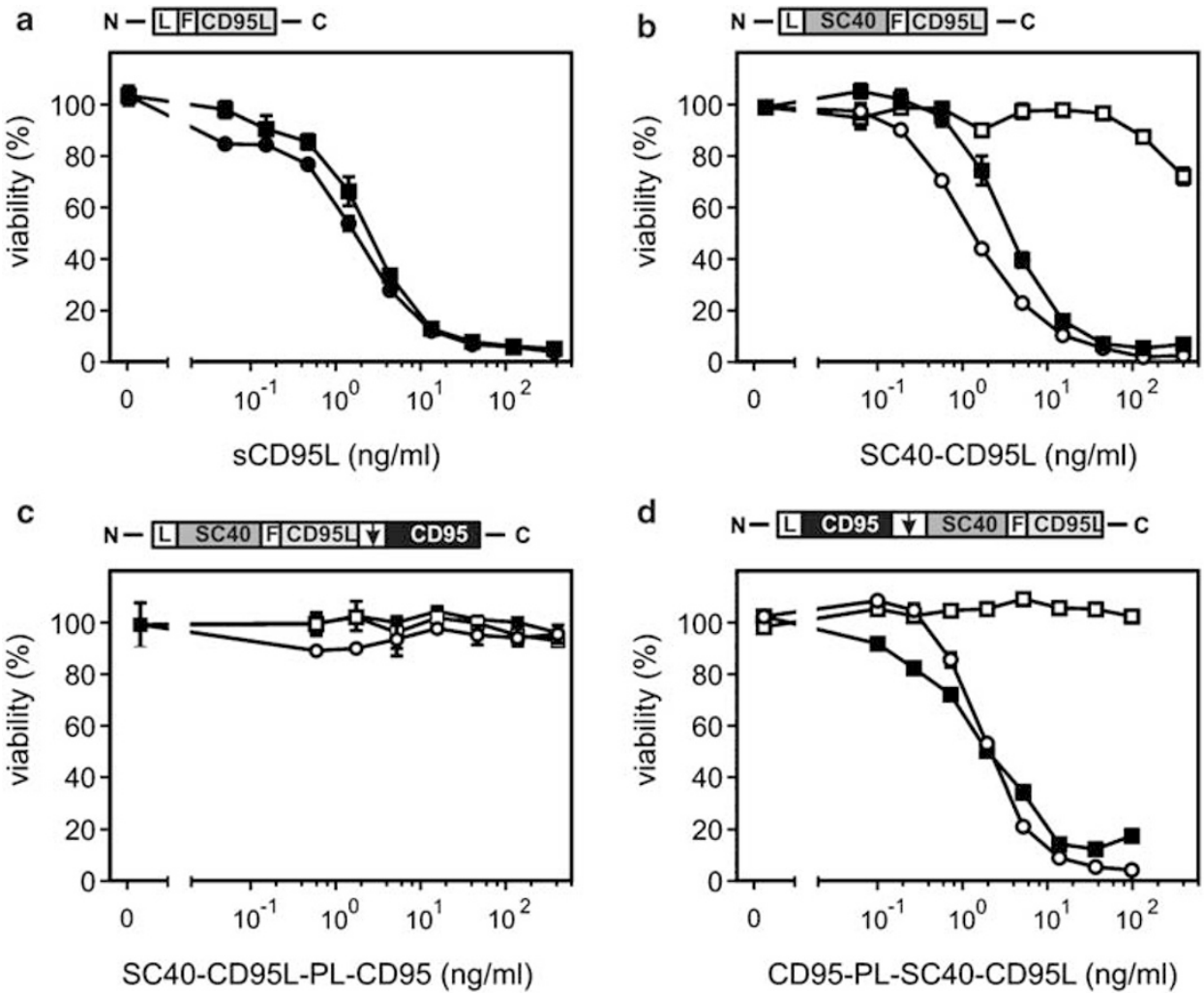

d

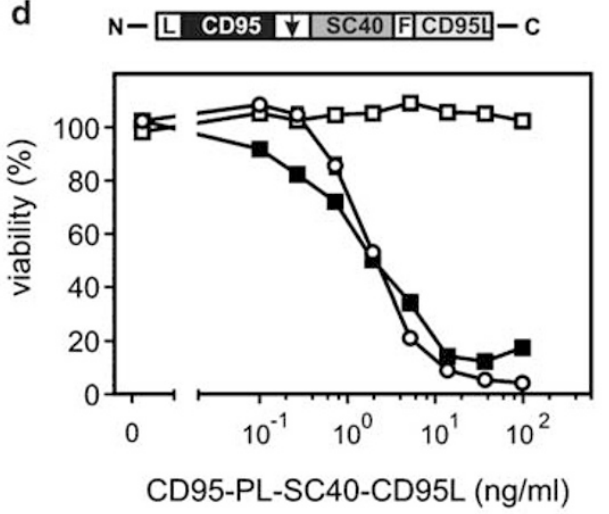

e
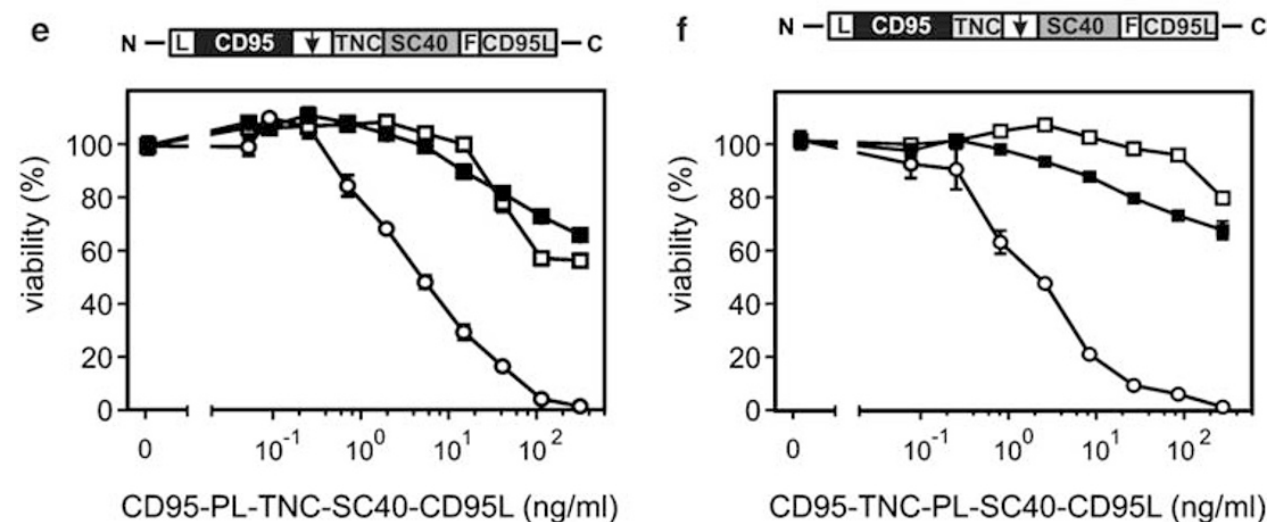

Figure 2 Cytotoxic activities of CD95L fusion proteins. (a-f) HT1080 (squares) and HT1080FAP cells (circles) were seeded in 96 -well plates (1.5 $\times 10^{4} \mathrm{cells} /$ well). Next day, cells were treated with $1 \mu \mathrm{g} / \mathrm{ml}$ cycloheximide and challenged in duplicates or triplicates with increasing concentrations of soluble CD95L (a) or the indicated CD95L fusion proteins (b-f) in the presence (filled symbols) or absence (open symbols) of $1 \mu \mathrm{g} / \mathrm{ml}$ of the crosslinking anti-Flag mAb M2. After additional $16 \mathrm{~h}$, cell viability was determined by crystal violet staining

less background activity after crosslinking (Figure 2) and better specific binding to the FAP antigen (Figure 3) than the CD95-PL-TNC-SC40-CD95L variant, we decided to concentrate subsequent analyses on the CD95-TNC-PL-SC40CD95L prodrug. For simplicity, this prodrug will be designated in the following SC40-CD95L-PD. As expected, SC40CD95L-PD also induced, in a FAP-dependent manner, nonapoptotic CD95L signaling (IL8 induction) when apoptosis induction was blocked by caspase inhibitors (Figure 4).

Activation of the CD95L prodrug SC40-CD95L-PD is dependent on FAP binding and MMP-mediated processing. HT1080 cells and its transfected derivative
HT1080FAP display comparable levels of endogeneous MMP activity, ${ }^{7}$ confirmed by gelatin zymography of wholecell lysates (data not shown). In accordance with the expected targeting dependent proteolytic activation, processed SC40-CD95L-PD was detectable in HT1080FAP cell cultures, but not in HT1080 cell cultures (Figure 5a, left panel). A crucial role of MMPs in FAP-dependent processing of SC40-CD95L-PD is suggested from blocking cleavage of SC40-CD95L-PD by treatment with the MMP inhibitor llomastat (Figure 5a, left panel). Moreover, complete processing was achieved by prodrug incubation with recombinant MMP2 (Figure 5a, right panel). Consistent with FAP-dependent processing and activation of SC40- 


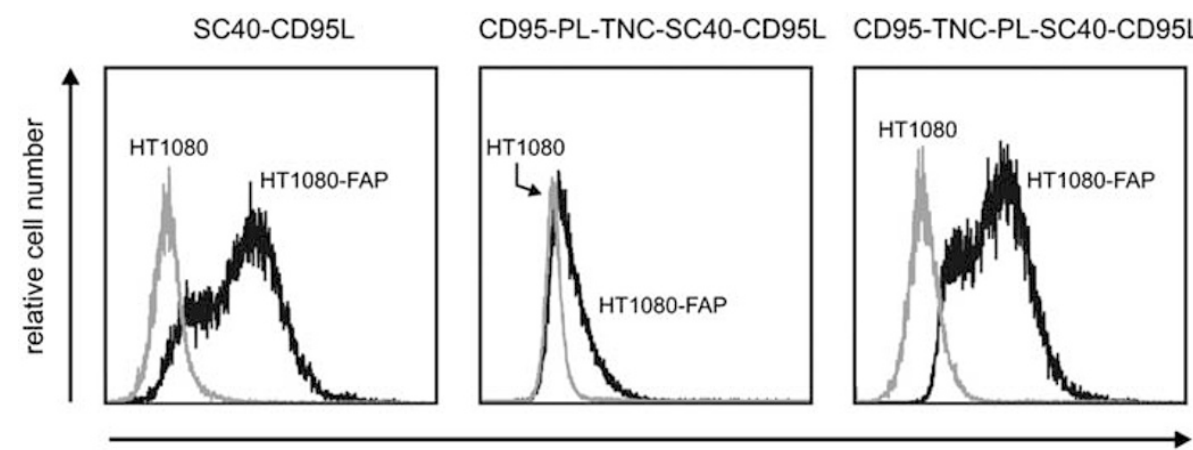

log fluorescence intensity

Figure 3 FAP binding of CD95L fusion proteins. HT1080 and HT1080FAP cells were incubated on ice with $5 \mu \mathrm{g} / \mathrm{ml}$ of the indicated fusion proteins and after repeated washing, bound proteins were detected by FACS using anti-Flag mAb M2 and FITC-labeled anti-mouse IgG

HT1080

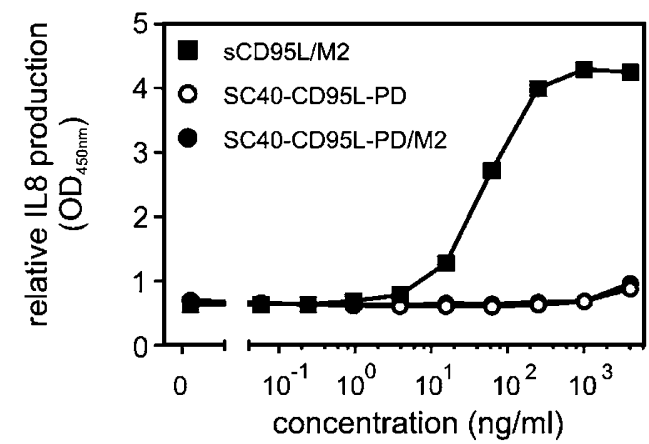

HT1080-FAP

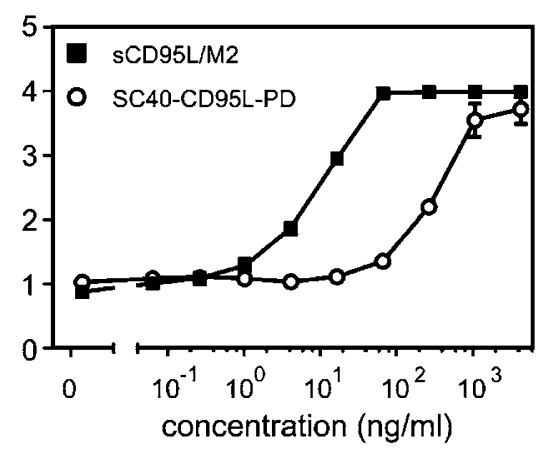

Figure 4 FAP-dependent stimulation of non-apoptotic CD95L signaling by SC40-CD95L-PD. HT1080 and HT1080FAP cells were seeded in 96-well plates. Next day, medium was replaced by culture medium containing $\mathrm{ZVAD}(20 \mu \mathrm{M})$ and cells were stimulated in triplicates with the indicated concentrations of the prodrug, M2-crosslinked prodrug or M2-crosslinked SCD95L. After $4 \mathrm{~h}$, supernatants were removed and their relative IL8 content was determined by ELISA

CD95L-PD, apoptosis induction by this prodrug was almost completely blocked on FAP-expressing cells by either neutralizing CD95L antibodies, neutralizing FAP-binding minibodies or by treatment with llomastat, respectively (Figure 5b). Autoinhibition of the SC40-CD95L-PD might be due to intermolecular or intramolecular interaction of the inhibitory CD95 domain and the CD95L domain. To distinguish between these two possibilities, we analyzed the SC40-CD965L-PD prodrug by chemical crosslinking and gel filtration. SDS-PAGE analysis followed by anti-Flag Western Blot under reducing and non-reducing conditions confirmed that the SC40-CD95L-PD formed covalently associated trimers as expected from the introduction of the tenascin-C trimerization domain (Figure 6a). ${ }^{12}$ Chemical crosslinking experiments of purified SC40-CD95L-PD with the homobifunctional crosslinker $\mathrm{BS}^{3}$ allowed the identification of prodrug multimeres up to hexameres (Figure 6b, arrowheads). In accordance with a hexameric organization of the SC40-CD95L-PD, gel filtration experiments and native polycarylamide gel electrophoresis revealed a native molecular mass of the prodrug of about $500 \mathrm{kDa}$ (Figure 6c and data not shown). Together, these data suggest that the SC40-CD95L-PD prodrug could assemble into head to tail dimers of covalently associated prodrug trimers. Accordingly, although the trimeric organization of the molecule is caused by the trimerization domain of TNC and the intrinsic trimerization tendency of the CD95L domain, the secondary dimerization of the trimers is driven by intermolecular interaction of a trimeric CD95L domain and a trimeric CD95 inhibitory domain of two different trimers (Figure 6d). Besides MMPs, HT1080 cells also express the tumor-associated protease urokinase-type plasminogen activator (UPA). We therefore tested the prodrug properties of a SC40-CD95L-PD variant in which the MMP2-sensitive linker has been replaced by an UPAsensitive linker. This prodrug was also processed and activated in an FAP-dependent manner (data not shown). Moreover, prodrug processing and activation were significantly attenuated by the UPA inhibitor amiloride, but not by llomastat, pointing to specific processing by HT1080associated uPA (data not shown).

The CD95L prodrug design is also applicable to selfaggregating single-chain fragments. Like soluble trimeric CD95L, trimeric scFv-CD95L fusion proteins stimulate CD95 up to 1000 -fold less efficient than their corresponding secondarily aggregated counterparts, but the fusion proteins can be converted to membrane CD95L-like, highly active CD95 stimulating entities by binding to a cell surface antigen. ${ }^{6}$ In principle, this allows antigen-restricted and therefore local activation of CD95 in vivo. However, this approach does not work with scFv molecules displaying an 

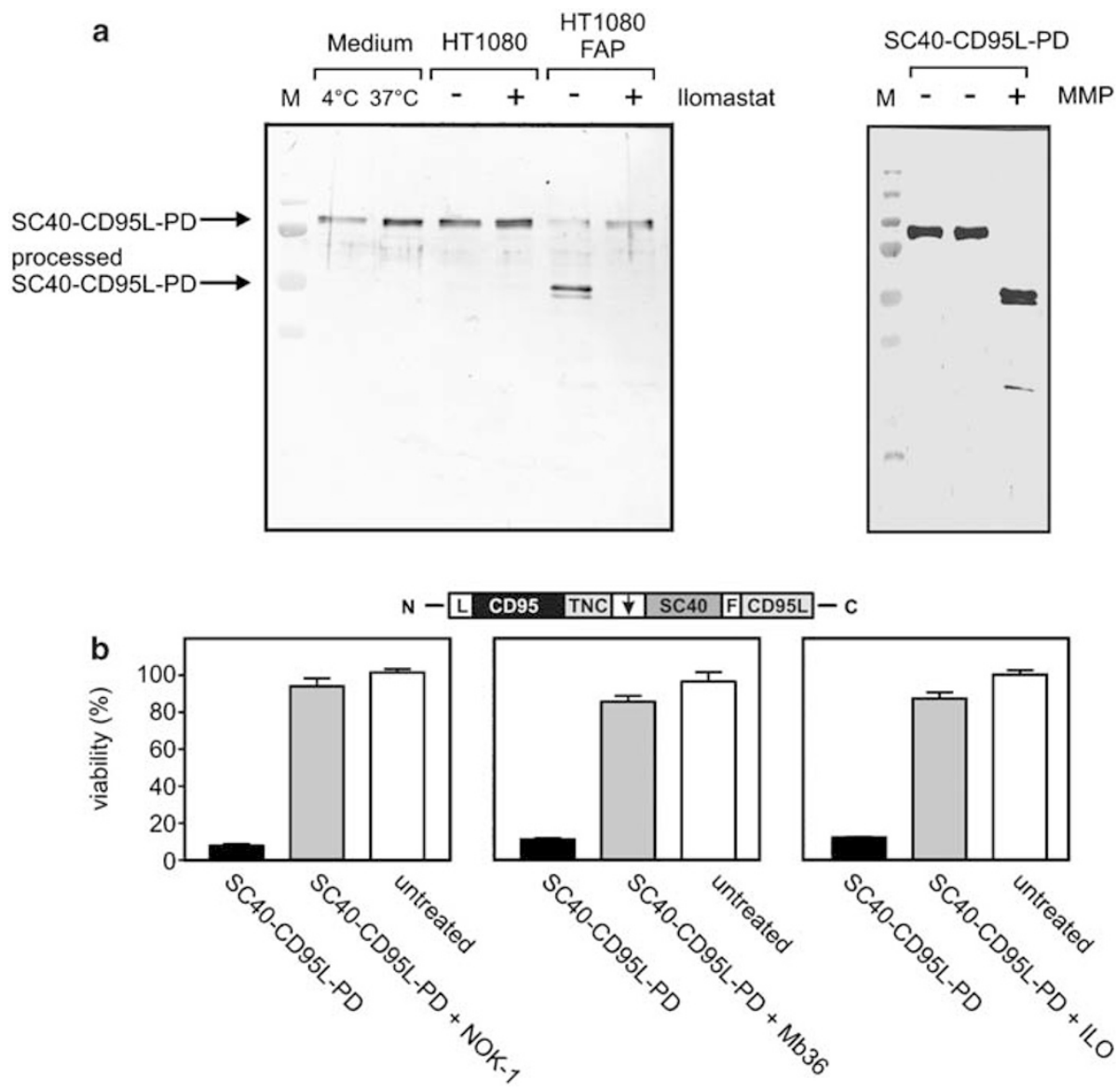

Figure 5 SC40-CD95L-PD-induced apoptosis occurs via CD95 and is dependent on FAP expression and MMP activity. (a) left panel, HT1080 and HT1080FAP cells were incubated for $16 \mathrm{~h}$ with SC40-CD95L-PD in the presence and absence of the MMP-specific inhibitor llomastat $(25 \mu \mathrm{M})$ and supernatants were analyzed by anti-Flag Western Blot; right panel, SC40-CD95L-PD $(12 \mu \mathrm{g} / \mathrm{ml})$ was incubated with recombinant MMP2 $(2 \mu \mathrm{g} / \mathrm{ml})$ in reaction buffer for $6 \mathrm{~h}$ at $37 \mathrm{C}$, aliquots were separated by SDS-PAGE and analyzed for processing by Western blotting using the Flag tag-specific monoclonal antibody M2. M = molecular weight standard. (b) HT1080FAP cells were seeded in 96-well plates and challenged the next day in duplicates with CD95L-PD $(15 \mathrm{ng} / \mathrm{ml})$. Where indicated, cells were pretreated with the CD95L-neutralizing mAb NOK-1 $(1 \mu \mathrm{g} / \mathrm{ml}$, left panel), with the FAP-blocking MB36 (10 $\mu \mathrm{g} / \mathrm{ml}$, middle panel) or with the MMP inhibitor llomastat (ILO, $25 \mu \mathrm{M}$, right panel)

intrinsic aggregation tendency as the latter leads to secondary aggregation, thereby targeting independent activation of the CD95L module within the corresponding fusion protein. The CD95L prodrug concept outlined above could therefore provide a mean also allowing the use of such scFv molecules for safe targeting of CD95. We therefore constructed and analyzed a CD95L prodrug, in which the scFv SC40, displaying no tendency to autoaggregate, has been replaced by a ScFv (SC36) of the same specificity (FAP), but with known autoaggregation. ${ }^{13}$ Indeed, a SC36CD95L fusion protein displayed a high activity to stimulate CD95 also on FAP-negative cells (Figure 7a, left panel). The corresponding SC36-derived CD95L prodrug (SC36-CD05L$P D)$, however, showed no activity on FAP-negative cells, but strongly activated CD95 on HT1080FAP cells (Figure 7a, right panel). Accordingly, processing of SC36-CD95L prodrug could only be detected upon incubation with FAPexpressing, but not parental, antigen-negative HT1080 cells (Figure 7b). The MMP inhibitor llomastat blocked cleavage of SC36-CD95L-PD by HT1080FAP cells, confirming specific action of MMPs (Figure 7b). The SC36-CD95L prodrug induced apoptosis on FAP-expressing HT1080 cells via
CD95 in an FAP- and MMP-dependent manner, evident from blocking of cell death by CD95L neutralizing NOK-1 mAb, FAP-neutralizing ScFv SC36 and MMP inhibitor llomastat, respectively (Figure 7c). Thus, the prodrug design described above allows usage of scFvs as targeting modules which exert an intrinsic tendency to autoaggregate.

Processing of a CD95L prodrug results in the release of the trimeric CD95-TNC part of the molecule, which represents a CD95L-neutralizing reagent. To analyze the extent to which the latter interferes with the CD95-stimulating action of the processed, that is, active SC36-CD95L part of the prodrug, we analyzed the neutralization capacity of recombinant Flagtagged CD95-TNC, resembling the cleaved off inhibitor fragment, in a SC36-CD95L-induced cell death assay on FAP-positive cells. We only found a significant inhibitory effect of Flag-CD95-TNC on FAP-dependent SC36-CD95L-induced apoptosis with a large excess (several 100-fold molar ratio) of Flag-CD95-TNC over SC36-CD95L (Figure 7d and e). As prodrug processing always yields stoichiometrically equal amounts of the CD95-TNC and SC36-CD95L part, no relevant neutralization effect should thus occur through prodrug processing-derived CD95-TNC. The low neutralization 

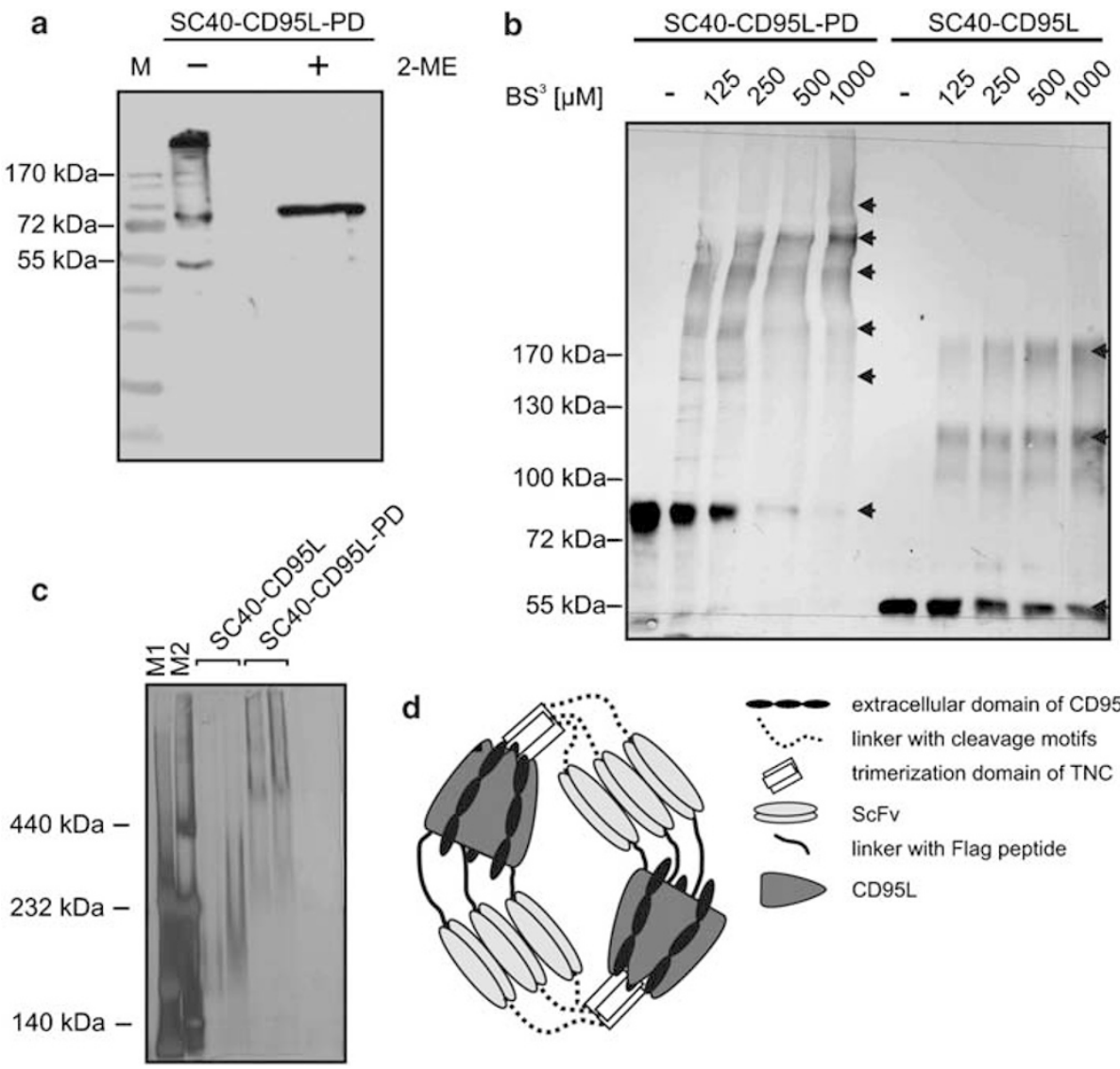

extracellular domain of CD95 linker with cleavage motifs trimerization domain of TNC ScFv linker with Flag peptide CD95L

Figure 6 Characterization of SC40-CD95L-PD. (a) SC40-CD95L-PD was separated under reducing and non-reducing conditions by SDS-PAGE and visualized by Western blot with the anti-Flag antibody M2 and alkaline phosphatase-labeled secondary anti-mouse rabbit lgG. M = molecular weight standard. (b) SC40-CD95L-PD was treated with the indicated concentrations of the chemical crosslinker $\mathrm{BS}^{3}$ for $30 \mathrm{~min}$ on ice. Samples were subsequently analyzed under reducing conditions by Western blot. Arrow heads indicate the monomer and several crosslinked products corresponding to dimers, trimers and higher molecular weight forms. (c) The SC40-CD95L fusion protein and the corresponding prodrug (SC40-CD95L-PD) in duplicates and two different molecular weight standards (M1, M2) were resolved by native polyacrylamide gel electrophoresis. Proteins were finally visualized by anti-Flag Western blot analysis. (d) Proposed organization of SC40-CD95L-PD hexamers

capacity of the CD95-TNC fusion protein, although better than those of the straight CD95 extracellular domain (CD95ed) or a CD95ed-Fc fusion protein (data not shown) is in good accordance with literature data obtained with a trimeric CD95 fusion protein with CMP. ${ }^{11}$ The fact, that the CD95-TNC part within the prodrug can efficiently neutralize the prodrug CD95L domain, is therefore likely related to the head to tail dimerization of two prodrug trimers which brings 'aviditiy' and/ or to the covalent linkage between both domains. We further verified on FAP-negative HT1080 cells whether the CD95L domain of the non-processed prodrug interferes with CD95Lmediated activation of CD95. Therefore, apoptosis was induced by a highly active Fc fusion protein of CD95L in the presence of the SC36-CD95L-PD prodrug. The latter showed at best a marginal inhibitory effect on Fc-CD95L-induced cell death, when the prodrug was given with greater than 100 -fold excess (Figure 7f).

The prodrug SC36-CD95L-PD inhibits development of FAP-positive tumors in nude mice. In order to probe the applicability of CD95L prodrugs and to verify target celldependent processing and activation in vivo, we analyzed systemic toxicity and antitumoral activity in several independent experiments. After intravenous (i.v.) injection of $80 \mu \mathrm{g} \mathrm{SC36-CD95L-PD,} \mathrm{all} \mathrm{mice} \mathrm{survived} \mathrm{the} 6 \mathrm{~h}$ observation period (Table 1) and showed at this time point neither caspase-3 activity in liver tissue nor increased transaminase levels in serum (Figure $8 a$ and b). In contrast, all mice treated with the corresponding SC36CD95L fusion protein died within $3.5 \mathrm{~h}$ similar to all mice that received crosslinked soluble CD95L as positive control (Table 1). Accordingly, mice treated with crosslinked CD95L displayed highly increased caspase-3 activity in liver tissue and showed high transaminase levels in the serum (Figure 8a and b). These data verified that the CD95L prodrug lacks acute systemic toxicity.

Antitumoral activity of SC36-CD95L-PD was subsequently analyzed in a tumor xenograft model using HT1080 or FAPexpressing HT1080 cells transplanted subcutaneous (s.c.) into the hind flank of NMRI nu/nu mice. Treatment with the prodrug commenced with two injections on the following day and two consecutive daily injections each with $50 \mu \mathrm{g}$ of SC36CD95L-PD given s.c. close to the site of tumor cell transplantation and tumor growth was monitored for 21 days. We observed significantly $(P<0.001)$ reduced tumor growth in the CD95L prodrug-treated group (Figure $8 \mathrm{c}$ and d), with 
a

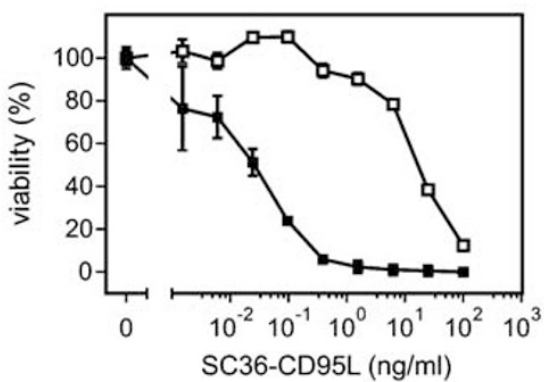

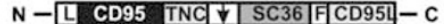

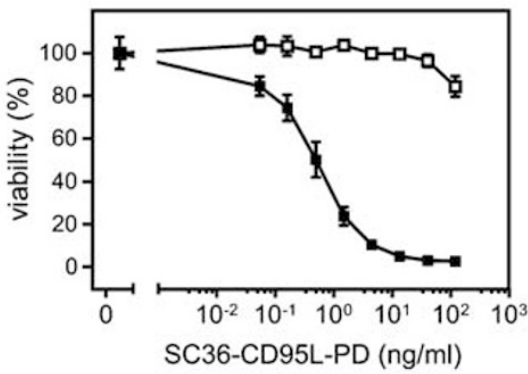

b

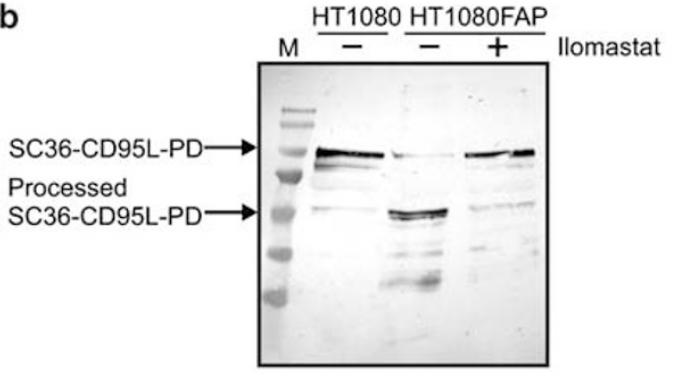

C

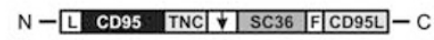
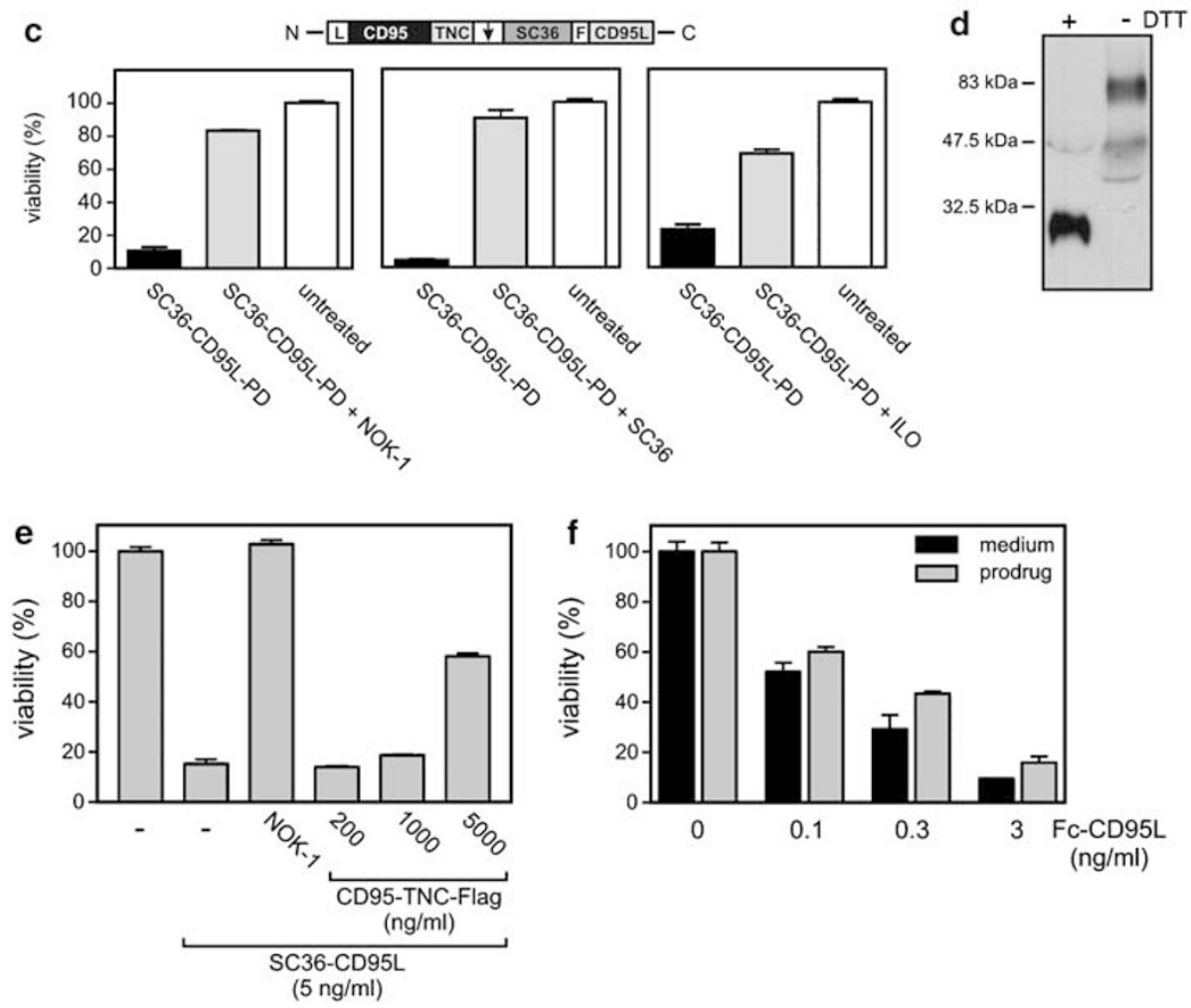

Figure 7 SC36-CD95L-PD-induced apoptosis occurs via CD95 and is dependent on FAP expression and MMP activity. (a) Triplicates of HT1080 (open squares) and HT1080FAP cells (filled squares) were incubated for $16 \mathrm{~h}$ in the presence of $\mathrm{CHX}(1 \mu \mathrm{g} / \mathrm{ml}$ ) with the indicated concentrations of SC36-CD95L (left panel) and SC36-CD95L-PD (right panel). Cell viability was finally determined by crystal violet staining. (b) HT1080 and HT1080FAP cells were incubated for 16 h with SC36-CD95L-PD in the presence and absence of the MMP-specific inhibitor llomastat $(25 \mu \mathrm{M})$ and supernatants were then analyzed by anti-Flag Western blot. (c) HT1080FAP cells were seeded in 96-well plates and challenged the next day with SC36-CD95L-PD $(5 \mathrm{ng} / \mathrm{ml})$ in the presence or absence of the CD95L-neutralizing mAb NOK-1 (1 $\mu \mathrm{g} / \mathrm{ml}$, left panel), the FAPneutralizing scFv SC36 $(50 \mu \mathrm{g} / \mathrm{ml}$, middle panel) or the MMP inhibitor llomastat $(25 \mu \mathrm{M}$, right panel). After additional $16 \mathrm{~h}$, cell viability was determined by crystal violet staining. (d) A carboxy-terminally Flag-tagged fusion protein comprising the CD95-TNC part of the SC36-CD95L-PD was produced in HEK293 cells and analyzed under reducing and non-reducing conditions by SDS-PAGE and anti-Flag Western blotting. (e) HT1080FAP cells were challenged for $16 \mathrm{~h}$ with SC36-CD95L (5 ng/ml), which was previously incubated for $1 \mathrm{~h}$ with the indicated concentration of CD95-TNC-Flag or $1 \mu / \mathrm{ml} \mathrm{NOK}-1$ in the presence of $1 \mu \mathrm{g} / \mathrm{ml} \mathrm{CHX}$. Finally, cell viability was determined by crystal violet staining. (f) HT1080 cells preincubated or not for $30 \mathrm{~min}$ with $300 \mathrm{ng} / \mathrm{ml}$ of SC36-CD95L-PD were challenged for $16 \mathrm{~h}$ with the indicated concentration of Fc-CD95L and then analyzed for cell viability by crystal violet staining 
five of eight of the animals staying tumor free until the end of the observation period ( 5 weeks) and two of the three tumorbearing animals showing either a barely detectable tumor or at least a clearly reduced tumor growth as compared to the control group (Figure $8 \mathrm{c}$ and d). In contrast, target-negative tumor cells showed $100 \%$ tumor take for both, the PBS control and the CD95L prodrug group. These data are in accordance with two additional experiments (data not shown) in which

Table 1 SC36-CD95L-PD shows no systemic toxicity in mice Treatment (i.v. injection) Surviving animals $\begin{gathered}\text { Animals dead } \\ \text { within } 6 \mathrm{~h}\end{gathered}$

\begin{tabular}{llr}
\hline $10 \mu \mathrm{g} \mathrm{sCD} 95 \mathrm{~L}^{\mathrm{a}} 10 \mu \mathrm{g} \mathrm{M} 2$ & 0 & $3^{\mathrm{b}}$ \\
$50 \mu \mathrm{g}$ SC36-CD95L & 0 & $3^{\mathrm{c}}$ \\
$80 \mu \mathrm{g}$ SC36-CD95L-PD & 3 & 0 \\
PBS & 3 & 0
\end{tabular}

i.v., intravenous; PBS, phosphate-buffered saline. $\mathrm{C} 57 \mathrm{BL} / 6 \times \mathrm{CBA} / \mathrm{J}$ mice received a single i.v. injection of the indicated fusion proteins. SC36-CD95L and the corresponding prodrug were applied in equivalent molarities. ${ }^{a} \mathrm{~A}$ second i.v. injection was given within 30 min after the injection of CD95L containing a Flag epitope allowing secondary crosslinking with the Flag-specific mAb M2. ${ }^{b}$ Mice died within $1 \mathrm{~h}$. ${ }^{\mathrm{C}}$ Mice died within $3.5 \mathrm{~h}$ mice were treated with $30 \mu \mathrm{g}$ of SC36-CD95L-PD for three consecutive daily injections (first experiment, 4-5 animals per group) or two times in a $9 \mathrm{~h}$ interval followed by two further daily injections (second experiment, eight animals per group), also showing prevention of tumor growth in 40 and $50 \%$ of animals transplanted with FAP-expressing cells, respectively. In these experiments, there was again 100\% tumor growth in all control groups (PBS treated, FAP-negative tumors; data not shown). Of note, in these experiments, the tumor-free animals were observed for a total of 2 months and stayed tumor free during this observation period.

In principle, CD95 stimulation in HT1080 cells induces apoptosis and IL8 production, whereby sensitization with $\mathrm{CHX}$ shifts the response towards apoptosis and treatment with ZVAD towards IL8 production (Figure 2 and 4). Many studies have shown that CD95-induced production of chemokines including IL8 can result in recruitment of neutrophils and tumor rejection (for a review, see Wajant, $2006^{14}$ ). The animal model investigated here was set to measure effects on tumor take and thus tumor cell transplanted mice were treated in an early phase, where tumors were not readily accessible for histological analysis. Therefore, we cannot conclude from
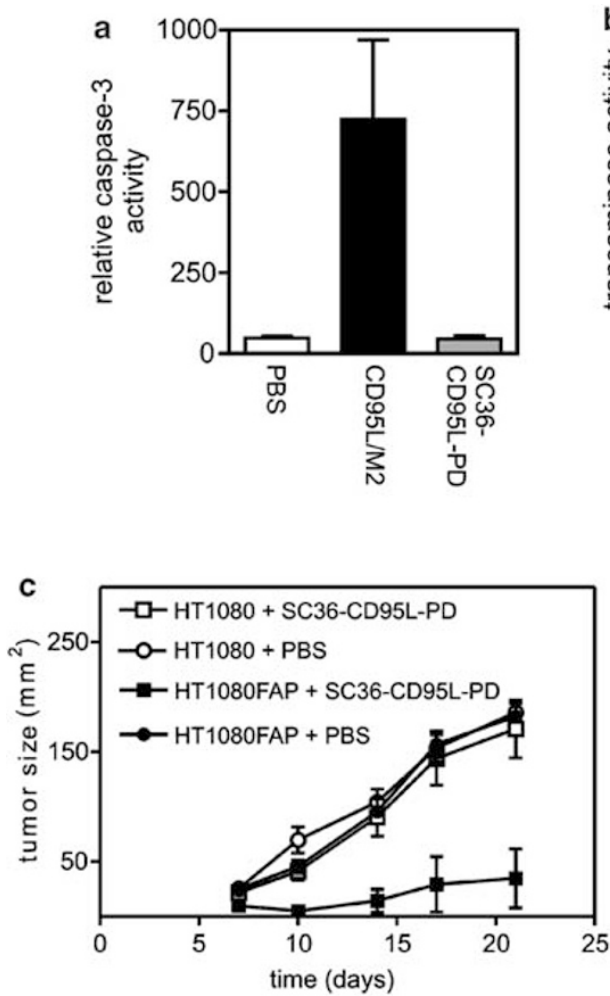
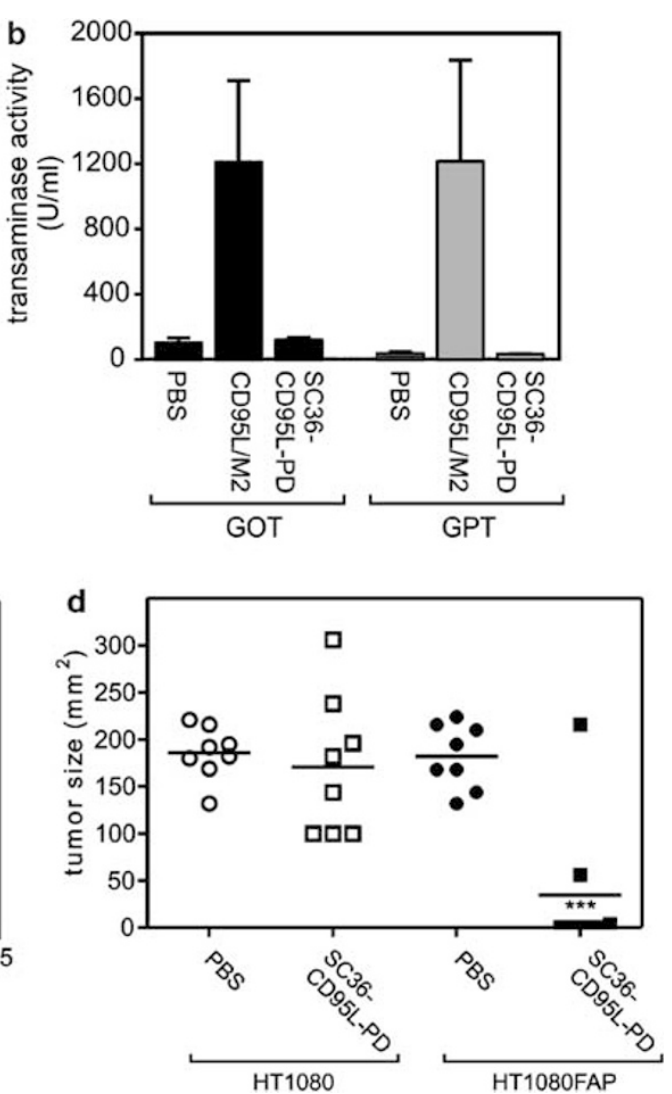

Figure 8 Analyses of systemic toxicity and antitumoral activity of SC36-CD95L-PD. (a, b) C57BL/6xCBA/J mice received a single i.v. injection of $80 \mu \mathrm{g} \mathrm{SC36-CD95L-PD}$ or as a positive control for CD95 activation, $10 \mu \mathrm{g}$ of soluble Flag-tagged CD95L followed by $10 \mu \mathrm{g}$ of the Flag tag-specific mAb M2 to allow formation of highly active secondary complexes of soluble CD95L. Liver tissues were removed either directly after death or $6 \mathrm{~h}$ after injection and analyzed for caspase-3 activity using the fluorigenic substrate Ac-DEVD-AMC (a). In parallel, serum was collected and analyzed with respect to the activity of glutamic oxaloacetic transaminase (GOT) and glutamic pyruvic transaminase (GPT) (b) $1.5 \times 10^{6}$ parental and FAP-expressing HT1080 cells were injected s.c. into the back of NMRI nu/nu mice and treatment was started 1 day later. Mice received four consecutive s.c. injections with $50 \mu \mathrm{g} \mathrm{SC36-CD95L-PD} \mathrm{close} \mathrm{to} \mathrm{the} \mathrm{site} \mathrm{of} \mathrm{tumor} \mathrm{cell} \mathrm{transplantation.} \mathrm{On} \mathrm{day} 1$ after tumor cell injection, mice were treated twice, followed by daily single treatment on days 2 and 3. Tumor growth was monitored as described. Data shown are the mean \pm S.E.M. of tumor sizes of eight animals/group as a function of time (c) and tumor size of the individual mice at day 21 with bars indicating the mean (d). ${ }^{* * *} P$-value $=0.0002$ compared to PBS-treated HT1080FAP-bearing animals 
this model on the relative contribution of direct tumor cell apoptosis induction versus stimulation of the innate immune system for the antitumoral effect of the CD95L prodrug on FAP-positive tumors.

In conclusion, we show that by genetic engineering a protein prodrug of CD95L can be generated that fulfills the essential criteria of a target restricted, antitumoral action. Our data prompt the following two-step model of CD95L prodrug activation and CD95 stimulation of target antigen- (FAP) expressing cells (Figure 9). First, the FAP-binding scFv of the prodrug traps this molecule on the cell surface of FAP-positive cells. Then, cell-associated MMPs/uPA cleaves the proteasesensitive linker between the inhibitory CD95 domain and the scFv-domain, allowing release by dissociation and/or further proteolytic degradation of the CD95 receptor domain. The unmasked scFV-CD95L module stays membrane bound and now mimics membrane CD95L, exerting a high signal capacity towards CD95-expressing cells in an autotropic and juxtatropic signaling mode. As tumor-selective activation of the prodrug is encompassed by tumor-associated proteases, we envisage that this principle can be applied for any cell surface molecule expressed by the tumor cells themselves, the tumor vasculature or stroma, as exemplified here using FAP as a model antigen. The animal studies provide first evidence for target-dependent processing and functional,

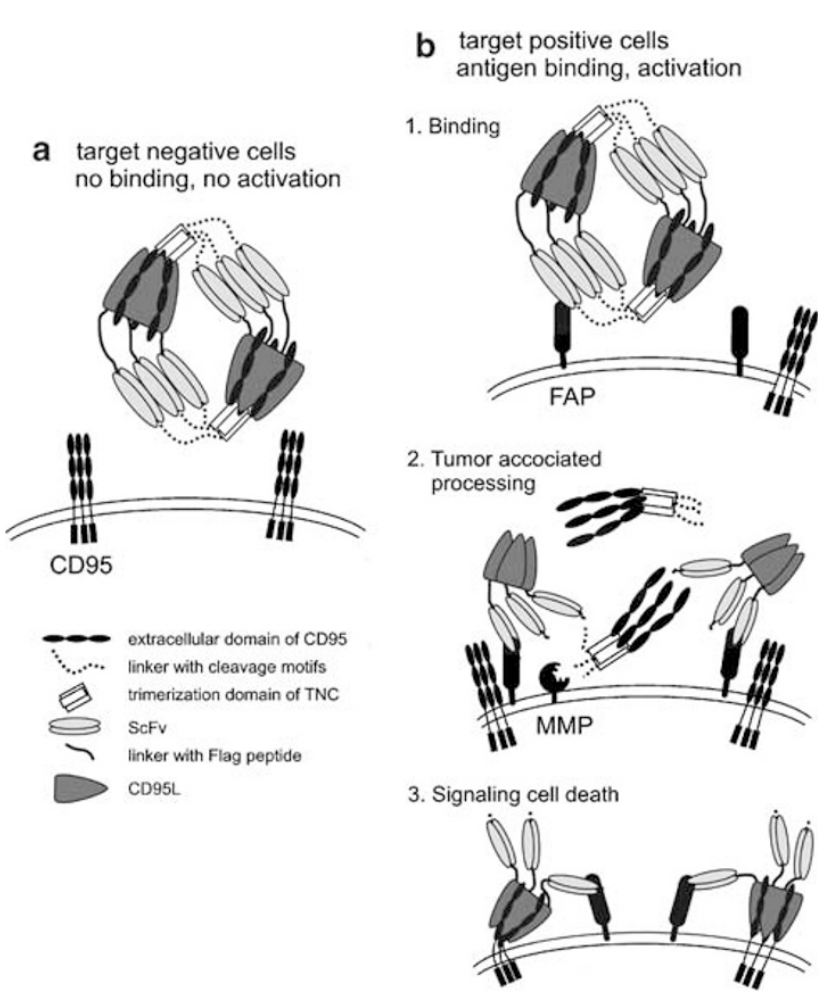

Figure 9 Model of FAP-dependent and MMP-mediated activation of an FAPspecific CD95L prodrug. The FAP-specific CD95L prodrug stays inactive on antigen-negative cells. On FAP-expressing cells, the scFv module of the prodrug traps this molecule on the cell surface. The still inactive CD95L prodrug can now be processed by cell surface-associated MMPs, leading to the release of the trimeric inhibitory CD95 domain. The unmasked, membrane-bound CD95L now mimics membrane CD95L exerting high signal capacity towards CD95-expressing cells tumor-restricted activation of the CD95L prodrug in vivo, warranting further studies on the systemic applicability, safety and antitumor efficacy of CD95L prodrugs.

\section{Materials and Methods}

Plasmids and cell lines. Using standard molecular cloning techniques, we engineered pCR3.1- (Invitrogen, Karlsruhe, Germany) derived expression plasmids encoding chimeric proteins that are build up as indicated in Figure 1 of in-framelinked cDNA fragments encoding a leader sequence, human and/or murine FAPspecific scFvs, a Flag tag, amino acids 139-281 of human CD95L, amino acids 15173 of human CD95, amino acids 110-139 of chicken tenascin-C and MMP2- or uPA-sensitive peptide linkers. The constructs were subcloned into the pIRESpuro3 vector (BD Biosciences, Clontech, Heidelberg, Germany) and finally the sequence of the chimeric cDNAs was confirmed by sequencing. HEK293 cells were obtained from the American Type Culture Collection (Manassas, VA, USA). The HT1080 and the FAP-expressing HT1080FAP cells were a kind gift of W. Rettig (Boehringer Ingelheim Pharma, Vienna, Austria). Cells were cultured in RPMI 1640 medium (Biochrom, Berlin, Germany) supplemented with $5 \%$ fetal calf serum (FCS). HT1080FAP cells were grown in the presence of $250 \mu \mathrm{g} / \mathrm{ml} \mathrm{G} 418$. Stably transfected cells expressing the fusion proteins were cultivated in the presence of $5 \mu \mathrm{g} / \mathrm{ml}$ puromycin.

Production and purification of recombinant proteins. The various CD95L fusion proteins were produced in HEK293 cells after transient transfection of the corresponding expression plasmids using lipofectamine 2000 (Invitrogen, Karlsruhe, Germany). After 3 days of cell culture in serum-free medium (Optimem, Invitrogen, Karlsruhe, Germany), supernatants were collected. Alternatively, HEK293 were stably transfected with the pIRESpuro3-derived expression constructs by selection with puromycin. For protein production, stable clones were expanded and grown to approximately $75 \%$ confluence in FCS-supplemented medium. FCS-containing medium was then removed and serum-free Optimem medium was added. Supernatants were collected after culture for 2-3 days and fusion proteins were purified by affinity chromatography on an anti-Flag M2 agarose column. After elution with PBS supplemented with $100 \mu \mathrm{M}$ Flag peptide (Sigma, Steinheim, Germany), protein-containing fractions were dialyzed against PBS and analyzed by SDS-PAGE.

Flow cytometry. HT1080 and HT1080FAP cells were incubated for $2 \mathrm{~h}$ at $4^{\circ} \mathrm{C}$ with $5 \mu \mathrm{g} / \mathrm{ml}$ of the indicated CD95L fusion protein. After washing three times with PBS, $0.2 \%$ FCS, $0.02 \%$ sodium azide, bound fusion proteins were detected by antiFlag $\mathrm{mAb}$ M2 $(1 \mu \mathrm{g} / \mathrm{ml}$ Sigma, Steinheim, Germany) and fluoresceine isothiocyanate-labeled rabbit anti-mouse IgG Ab $(1 \mu \mathrm{g} / \mathrm{ml}$ Sigma, Steinheim, Germany). Analyses were performed using EPICS-XL (Coulter, Krefeld, Germany) according to standard procedures.

Chemical crosslinking with $\mathbf{B S}^{3}$. The indicated CD95L fusion protein $(200 \mathrm{ng})$ was incubated with varying concentrations of the chemical crosslinker BS ${ }^{3}$ (Pierce, Rockford, USA) for 30 min on ice in a total volume of $18 \mu$ l. The crosslinking reaction was quenched by adding $2 \mu \mathrm{l} 1 \mathrm{M}$ Tris- $\mathrm{HCl}(\mathrm{pH}$ 7.5) and proteins were finally analyzed by SDS-PAGE and Western Blot.

Activation of CD95L prodrugs. For in vitro activation of prodrugs, the purified proteins $(12 \mu \mathrm{g} / \mathrm{ml})$ were incubated with $2 \mu \mathrm{g} / \mathrm{ml}$ recombinant MMP-2 (Merck Bioscience $\mathrm{GmbH}$, Schwalbach, Germany) in reaction buffer $\left(20 \mu \mathrm{M} \mathrm{ZnSO}_{4}\right.$, $1 \mathrm{mM} \mathrm{CaCl}, 0.05 \%$ Brij-35, $50 \mathrm{mM}$ Tris- $\mathrm{HCl}(\mathrm{pH} 7.5))$ for $6 \mathrm{~h}$ at $37^{\circ} \mathrm{C}$. Prodrug processing and activity were finally analyzed by SDS-PAGE, Western Blot and viability assay. To analyze cell-mediated processing of CD95L prodrugs, HT1080 and HT1080FAP cells $\left(2 \times 10^{4} /\right.$ well) were grown overnight in $100 \mu$ l culture medium in 96-well plates. The following day, medium was replaced by $100 \mu \mathrm{l}$ Optimem containing $10 \mu \mathrm{g} / \mathrm{ml}$ purified prodrug. After $16 \mathrm{~h}$ of incubation, the supernatant was centrifuged and further analyzed by Western Blot with anti-Flag Ab. To verify the specificity of processing, cells were preincubated with $25 \mu \mathrm{M}$ of the MMP inhibitor llomastat (Chemicon International, Hofheim, Germany). Molecular weight standards were obtained from Biorad (Munich, Germany and Fermentas, St. Leon-Rot, Germany).

Native gel electrophoresis. Four hundred nanograms of purified protein was separated by native gel electrophoresis in $1 \times$ Tris-Glycin running buffer 
Acknowledgements. This work was supported by Deutsche Krebshilfe (Grant 10-1751-Wa 3), Wilhelm-Sander-Stiftung (Grant 2003.120.1) and IZKF Wuerzburg (project B-32). We thank Dr Hans-Joachim Blum and Martina Schmitt (Medical Clinic and Polyclinic II, University of Wuerzburg) for performing transaminase assays, Markus Lautenbacher and Dafne Müller (both Institute of Cell Biology and Immunology, University of Stuttgart) for help in prodrug production and in animal treatment studies, respectively.

Apoptosis assays. HT1080 and HT1080FAP cells $\left(2 \times 10^{4} /\right.$ well) were grown overnight in $100 \mu$ l culture medium in 96-well plates. The following day, cells were challenged in duplicates or triplicates with the indicated concentrations of the various CD95L fusion proteins in the presence or absence of $1 \mu \mathrm{g} / \mathrm{ml} \mathrm{Flag-specific}$ $\mathrm{mAb} M 2$ and after $16 \mathrm{~h}$, cell viability was determined by crystal violet staining. Assays were performed in the presence of $1 \mu \mathrm{g} / \mathrm{ml}$ cycloheximide to sensitize cells for apoptosis induction. To demonstrate that apoptosis induction was dependent on CD95, CD95L fusion proteins were incubated with $1 \mu \mathrm{g} / \mathrm{ml}$ anti-CD95L NOK-1 (BD Biosciences, Heidelberg, Germany) prior stimulation. FAP-dependency of prodrug activation was shown by preincubation of the cells with $10 \mu \mathrm{g} / \mathrm{ml}$ of the recombinant anti-FAP minibody MB36 or $50 \mu \mathrm{g} / \mathrm{ml}$ of the corresponding recombinant scFv SC36.

IL8 ELISA. HT1080 and HT1080FAP cells $\left(1.5 \times 10^{4} /\right.$ well) were seeded in 96 well tissue culture plates and grown overnight. Next day, the medium was replaced by medium containing $20 \mu \mathrm{M} \mathrm{z-VAD}$ to avoid apoptosis induction and cells were stimulated in triplicates with the indicated concentrations of the CD95L prodrug. After $4 \mathrm{~h}$, supernatants were removed and analyzed using an IL8 ELISA kit (BD Biosciences, Heidelberg, Germany) according to the manufacturer's instructions.

Determination of caspase-3 and transaminase activities. To determine caspase-3 activity in the liver tissue of mice, homogenates were prepared in lysis buffer containing $200 \mathrm{mM} \mathrm{NaCl}, 20 \mathrm{mM}$ Tris, $1 \% \mathrm{NP}-40$ (pH 7.4). Thirty micrograms of protein was measured in triplicates and analyzed by conversion of the fluorogenic substrate Ac-DEVD-AMC (Alexis Biochemicals, Gruenback, Germany) according to the manufacturer's instructions. The activities of glutamic oxaloacetic transaminase (GOT/AST) and glutamic pyruvic transaminase (GPT/ALT) were determined with the AST and ALT determination kits of Roche (Mannheim, Germany) according to the manufacturer's recommendations.

Animal studies. Animal care and all experiments performed were in accordance with federal guidelines and have been approved by university and state authorities. S.c. tumor growth of parental $\mathrm{HT} 1080$ and FAP transfectants was assessed by s.c. injection of $1.5 \times 10^{6}$ cells suspended in $100 \mu \mathrm{l}$ of $0.9 \% \mathrm{NaCl}$ solution into the back of narcotized (Isofluran) NMRI nu/nu mice. Mice (eight animals/group) were treated on day 1 after tumor cell injection twice with $50 \mu \mathrm{g}$ SC36-CD95L-PD in $100 \mu \mathrm{l}$, followed by a daily single treatment at the same dose on days 2 and 3 in the area of tumor cell application. Control groups received $100 \mu \mathrm{l}$ PBS at the same time intervals. Tumor growth was monitored as described. ${ }^{15}$ For statistical analysis, two-tailed $P$-value was analyzed by unpaired $t$-test on day 21 . To investigate systemic toxicity of SC36-CD95L and the corresponding prodrug SC36CD95L-PD, groups of three C57BL6xCBA/J mice were treated with the indicated doses of the reagents by i.v. application. Survival and phenotypical signs of sickness were monitored for $6 \mathrm{~h}$ before mice were killed for determination of caspase-3 activity in liver tissues.
Debatin KM, Krammer PH. Death receptors in chemotherapy and cancer. Oncogene 2004;

2. Igney FH, Krammer PH. Tumor counterattack: fact or fiction? Cancer Immunol Immunother 2005; 54: 1127-1136.

3. Wajant H, Pfizenmaier K, Scheurich P. Non-apoptotic Fas signaling. Cytokine Growth Factor Rev 2003; 14: 53-66.

4. Wajant H, Gerspach J, Pfizenmaier K. Tumor therapeutics by design: targeting and activation of death receptors. Cytokine Growth Factor Rev 12005; 6: 55-76.

5. Schneider $\mathrm{P}$, Holler N, Bodmer JL, Hahne M, Frei K, Fontana $A$ et al. Conversion of membrane-bound Fas(CD95) ligand to its soluble form is associated with downregulation of its proapoptotic activity and loss of liver toxicity. J Exp Med 1998; 187: 1205-1213.

6. Samel D, Muller D, Gerspach J, Assohou-Luty C, Sass G, Tiegs G et al. Generation of a FasL-based proapoptotic fusion protein devoid of systemic 32077-32082.

7. Gerspach J, Muller D, Munkel S, Selchow O, Nemeth J, Noack M et al. Restoration of membrane TNF-like activity by cell surface targeting and matrix metalloproteinase-mediated processing of a TNF prodrug. Cell Death Differ 2006; 13 273-284.

8. Wuest T, Gerlach E, Banerjee D, Gerspach J, Moosmayer D, Pfizenmaier K. TNFselectokine: a novel prodrug generated for tumor targeting and site-specific activation of tumor necrosis factor. Oncogene 2002; 21: 4257-4265.

9. Schmidt A, Muller D, Mersmann M, Wuest T, Gerlach E, Garin-Chesa P et al. Generation of human high-affinity antibodies specific for the fibroblast activation protein by guided selection. Eur J Biochem 2001; 268: 1730-1738.

10. Garin-Chesa P, Old LJ, Rettig WJ. Cell surface glycoprotein of reactive stromal fibroblasts as a potential antibody target in human epithelial cancers. Proc Natl Acad Sci USA 1990; 87: 7235-7239.

11. Holler N, Kataoka T, Bodmer JL, Romero P, Romero J, Deperthes D et al. Development of improved soluble inhibitors of FasL and CD40L based on oligomerized receptors. $\mathrm{J}$ Immunol Methods 2000; 237: 159-173.

12. Kammerer RA, Schulthess T, Landwehr R, Lustig A, Fischer D, Engel J. Tenascin-C hexabrachion assembly is a sequential two-step process initiated by coiled-coil alphahelices. J Biol Chem 1998; 273: 10602-10608.

13. Brock B, Garin-Chesa P, Behrle E, Park JE, Rettig WJ, Pfizenmaier K et al. Speciescrossreactive scFv against the tumor stroma marker 'fibroblast activation protein' selected by phage display from an immunized FAP-/- knock-out mouse. Mol Med 2001; 7 461-469.

14. Wajant H. CD95L/FasL and TRAIL in tumour surveillance and cancer therapy. Cancer Treat Res 2006; 130: 141-165. efficacy of systemically administered recombinant tumor necrosis factor against several murine tumors in vivo. J Immunol 1987; 138: 963-974. 23: 2950-2966 toxicity due to cell-surface antigen-restricted activation. J Biol Chem 2003; 278 .

15. Asher A, Mule JJ, Reichert CM, Shiloni E, Rosenberg SA. Studies on the anti-tumor 